\title{
Electrical and Optical Characterization of the Plasma Needle for Use in Biomedical Applications
}

\author{
Biswajit Bora,,${ }^{a}{ }^{*}$ A. Aguilera, ${ }^{b}$ J. Moreno, ${ }^{a}$ J. Jain,,${ }^{a, c}$ C. Pavez, ${ }^{a}$ G. Avaria, ${ }^{a}$ M.J. \\ Inestrosa-Izurieta, ${ }^{a}$ S. Davis, ${ }^{a} \&$ L. Soto ${ }^{a}$ \\ aComisión Chilena de Energía Nuclear, Santiago, Chile; ' $U$ niversidad Metropolitana de Ciencias \\ de la Educación, Santiago, Chile; 'Universidad de Talca, Talca, Chile \\ *Address all correspondence to: Biswajit Bora, Comisión Chilena de Energía Nuclear, Santiago, Chile; Tel.: +56 22364 \\ 6244; Fax: +56 22364 6106, E-mail: bbora@cchen.cl or biswajit.cpp@gmail.com
}

\begin{abstract}
A plasma needle is a novel design of a plasma source at atmospheric pressure to achieve a nonthermal plasma jet. The advantage of the plasma needle is that it can be operated in open air, outside a vessel. The amount of plasma that is generated by a plasma needle is small $(\sim 1 \mathrm{~mm})$, and the plasma is nonthermal. Temperature of the neutral particles and ions is approximately at room temperature, and the particles can suitably interact with living biological cells without damaging the cells. In this work, we report on electrical characteristics and optical emission spectra (OES) of a plasma jet, produced by a direct-current plasma needle that easily interacts with living cells (a human finger). Argon gas is used to run the needle, and the plasma jet is operated on a water-covered agar-gel surface instead of the human finger for stability during characterization. The electrical diagnostics of the plasma needle show that the discharge pulsates. Increased applied voltage increases discharge frequency without affecting discharge voltage or discharge current. The frequency of the discharge is found to increase almost linearly with increasing applied voltage. Using a Boltzmann's plot method from the data collected with OES, we found the estimated excitation temperature was almost independent of the applied voltage.
\end{abstract}

KEY WORDS: plasma needle, plasma torch, electron temperature, electronic excitation temperature

\section{INTRODUCTION}

A plasma needle device produces nonthermal, atmospheric pressure, glow discharge plasma. With a single-electrode configuration, the novel design of the plasma source at atmospheric pressure makes it possible to achieve a nonthermal plasma jet. ${ }^{1-3}$ The advantage of a plasma needle is that it can be operated in open air, outside a vessel. The amount of plasma that is generated is small $(\sim 1 \mathrm{~mm})$, and the plasma is nonthermal. Temperature of the neutral particles and ions is approximately at room temperature, and the particles can suitably interact with living biological cells without damaging the cells. ${ }^{2-9}$ A plasma needle can be operated within a wide range of frequencies, including radio frequency, ${ }^{1,10}$ alternating current (AC), ${ }^{11-13}$ and DC (direct current). ${ }^{2}$ This type of plasma can operate near room temperature, allows treatment of irregular surfaces, and has a small penetration depth. These characteristics give the needle great potential for use in the biomedical field. 
Several experiments have shown that the plasma needle is capable of bacterial decontamination, localized cell removal without causing necrosis to treated or neighboring cells, and cancer-cell ablation. ${ }^{-7,9,14-19}$ It is believed that plasma particles, such as radicals and ions, and emitted ultraviolet light interact with the cell membranes and cell-adhesion molecules, causing cell detachment. ${ }^{4,21}$ Due to the plasma needle's nonequilibrium nature, the electron temperature of the plasma needle is quite high (measured in electron volts), but the temperature of heavy plasma particles such as ions and radical neutrals is at room temperature. High-temperature electrons can interact with and destroy living bacteria, microorganisms, or microbes without affecting the living cell/human body as a result of the low energy (due to the low mass of the electrons). We found that the characteristic of electron temperature is necessary for properly implementing, understanding, scaling, and optimizing atmospheric pressure plasma for specific biomedical investigation.

In this work, we report on the electrical characterization and optical emission spectra (OES) of a plasma needle that functions on a DC power supply and can interact with living cells (a human finger). Argon gas is used to run the plasma needle, and the plasma jet is operated on a water-covered agar-gel surface instead of the human finger, for stability during the characterization (assuming that the plasma properties do not change considerably on contact with a human body and the water-covered agar-gel surface). We estimated the excitation temperatures, which also indicate the tentative behavior of plasma electron temperature, by using the Boltzmann plot method from OES data. To measure plasma properties, we also investigated the influence of applied DC voltage, ballast resistance, and the separation between the tip of the needle and the water-covered agar-gel surface.

\section{EXPERIMENT}

The plasma needle is comprised of a metallic needle covered by a coaxial Teflon tube terminating with a metallic end. Several well-machined Teflon pieces are arranged to feed the gas and electrical connection. The plasma needle, its operation, and components are shown in Fig. 1. The needle is powered by a high-voltage $(0-15 \mathrm{kV}$, continuously adjustable) DC power supply through a ballast resistor (measured on the order of milliohms). A small amount of argon (delivered at a few milliliters per minute) passes through the hole of the metallic end to produce argon plasma. This helps to analyze the spectroscopic data. When a counter electrode such as a human finger or any other object is placed near the needle tip, plasma is produced. To characterize the plasma needle, the plasma jet is operated on a water-covered agar-gel surface for stability. An arrangement is made to adjust the separation between the tip of the plasma needle and the water-covered agar-gel surface. The current is measured by a set of Tektronix (Beaverton, OR) current probes (CT1, TCP 312A, and TCPA 300). Discharge voltages at the electrode are measured by a Tektronix voltage probe (P6015A). Voltage and current data are recorded with the help of a Tektronix oscilloscope (MDO 3034). The light from the plasma is collected by focusing a fiber optic cable and feeding it to an ASEQ Instruments (Vancouver, Canada) universal 


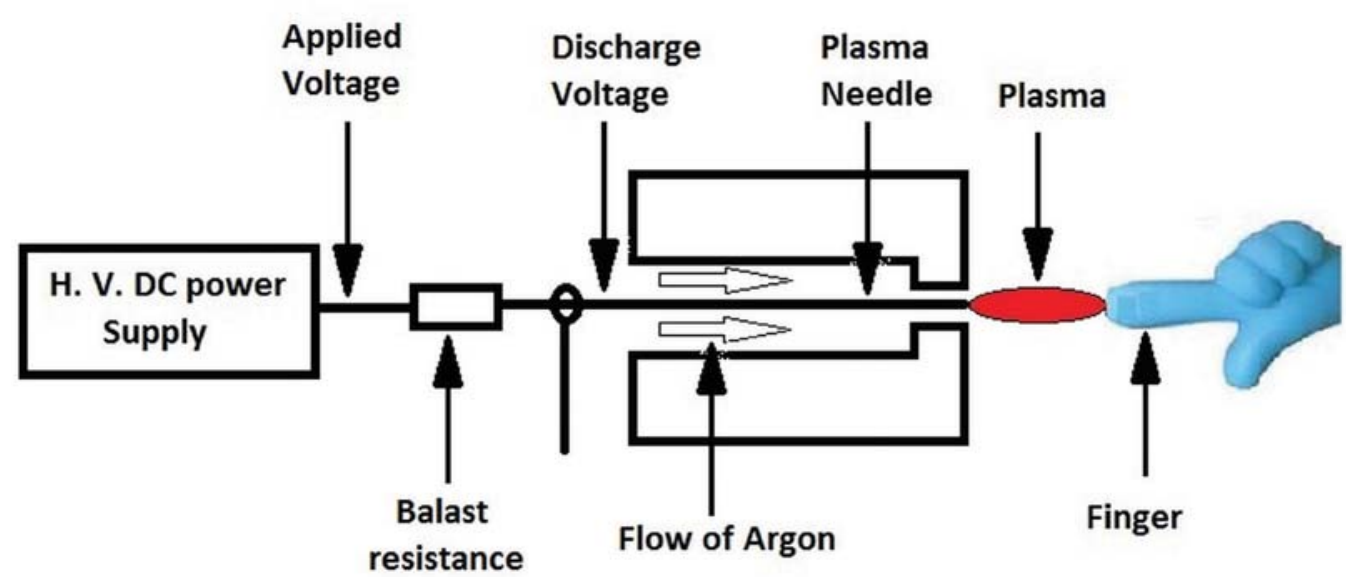

FIG. 1: Schematic of the plasma needle and its operation

serial bus spectrometer (model LR1). In this study, the applied DC voltage varied from 6.5 to $12.5 \mathrm{kV}$ and ballast resistance from 10 to $75 \mathrm{M} \Omega$. The separation between the tip of the needle and the water-covered agar-gel surface varied from 2 to $20 \mathrm{~mm}$.

\section{RESULTS AND DISCUSSION}

A typical image of the plasma jet interacting with a human finger is shown in Fig. 2(a). We found that the plasma jet can continually contact the human finger without the occurrence of any sensation. However, the plasma jet is operated on the water-covered agar-gel surface to mimic the interaction of the plasma with living cells and to illustrate the stability of the plasma jet (Fig. 2[b]).

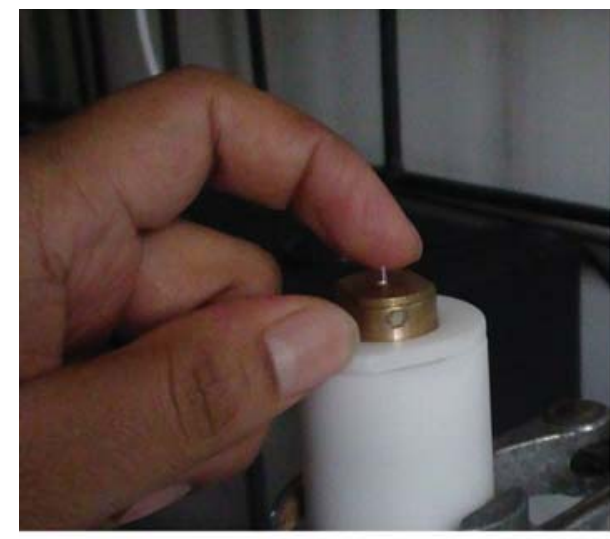

(a)

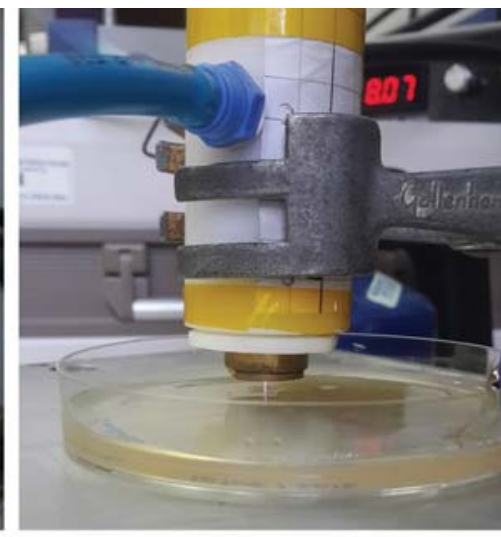

(b)

FIG. 2: Photographs of (a) the plasma jet interacting with a human finger and (b) the plasma jet operated on a water-covered agar-gel surface

Volume 7, Issue 4, 2017 
Figure 3 shows the electrical discharge characteristics of the plasma needle operating over the water-covered agar-gel surface, for an applied voltage of $10 \mathrm{kV}$. The electrical characteristics show that the discharge is pulsed in nature. We observed that increases in applied voltage augment the discharge frequency without considerably affecting discharge voltage and current. Discharge frequencies for different applied voltages are shown in Fig. 4. We found that discharge frequency increases linearly with increasing applied voltage. It is worth mentioning that the frequency of the discharge is almost independent of the applied voltage; however, a linear increment occurred below a threshold voltage. The observations in the present study agree with previously reported findings of liner increments of frequency that occur with increasing applied voltage below a threshold voltage. ${ }^{2}$

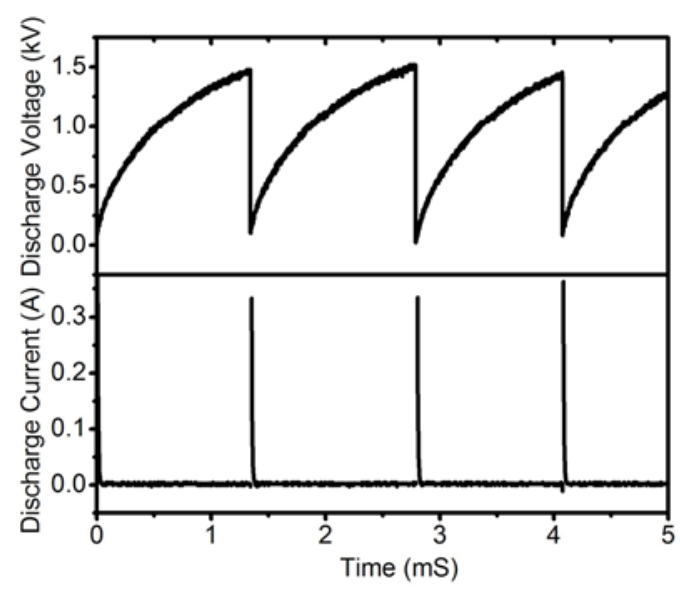

FIG. 3: Time evolution of the discharge current and discharge current of the plasma needle

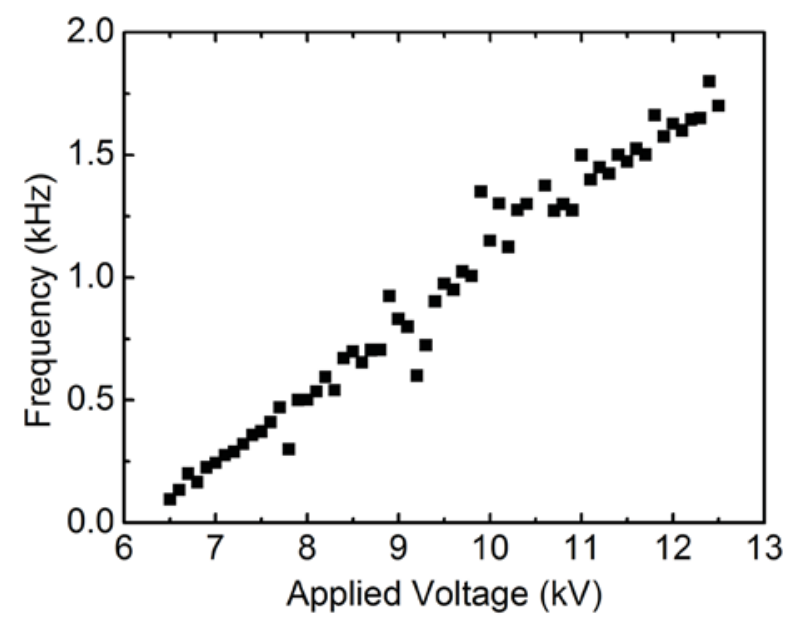

FIG. 4: Variation of discharge frequency with applied DC voltage 
Typical OES spectra from the plasma needle are shown in Fig. 5. We found the Ar II lines to be more prominent. Ar II lines at 289.16, 289.67, 303.35, 308.5, 309.34, 329.36, $329.29,330.72,347.67,351.43,370.69,379.04,394.6$, and $404.11 \mathrm{~nm}$ can be clearly seen in the spectra. However, the lines at 289.16 and $289.67 \mathrm{~nm}, 308.5$ and $309.34 \mathrm{~nm}$, and $329.36,329.29$, and $330.72 \mathrm{~nm}$ are superimposed to others due to the limitation of the spectrometer. For analysis of the OES spectra, those superimposed lines were ignored; we only considered the lines shown in Table 1.

On the basis of the emission spectral-line intensity, we were able to estimate excitation temperature of the plasma. Temperature could be calculated with either the intensity ratio of two lines or the Boltzmann plot method, and any error related to measurements were reduced by considering several lines using the Boltzmann plot method. ${ }^{22,23}$

Considering $I_{1}$ and $I_{2}$ to be the intensity of two different emissions at wavelengths $\lambda_{1}$ and $\lambda_{2}$, a ratio is given by ${ }^{19}$

$$
\frac{I_{1}}{I_{2}}=\frac{A_{1} g_{1} \lambda_{2}}{A_{2} g_{2} \lambda_{1}} \exp \left[\frac{E_{2}-E_{1}}{k T}\right],
$$

where $A$ is the Einstein coefficient of spontaneous emission (transition probability), $g$ is the statistical weight of the upper level of transition, $E$ is the energy of the upper level of

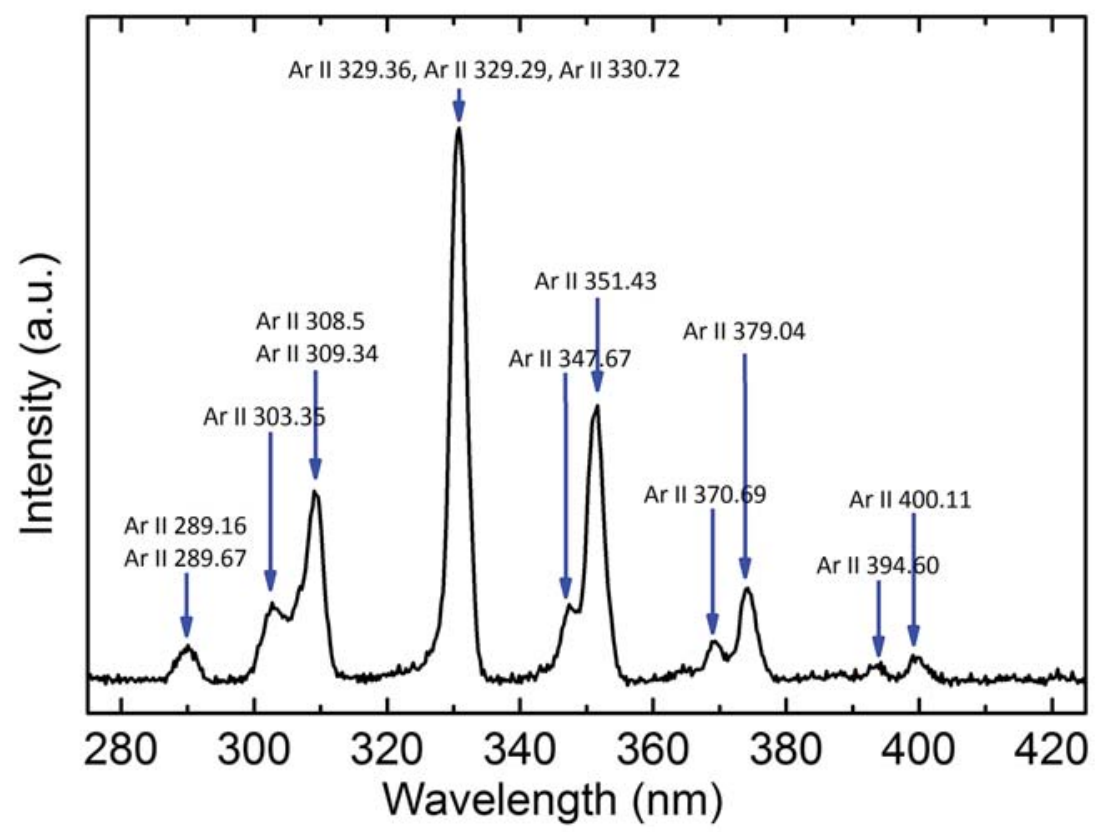

FIG. 5: Typical OES spectra of plasma produced by the plasma needle on a water-covered agargel surface. Ar, Argon

Volume 7, Issue 4, 2017 
TABLE 1: Details of the spectral lines used to estimate the excitation temperature from OES spectra

\begin{tabular}{lccc}
\hline Ion & Wavelength $(\mathbf{n m})$ & $\boldsymbol{A}_{\boldsymbol{k} i}\left(\mathbf{S}^{-1}\right)$ & $\boldsymbol{E}_{\boldsymbol{k}}\left(\mathbf{c m}^{-1}\right)$ \\
\hline Ar II & 303.35 & $9.9 \times 10^{6}$ & 172213.8798 \\
Ar II & 347.67 & $1.25 \times 10^{8}$ & 183797.4473 \\
Ar II & 351.43 & $1.36 \times 10^{8}$ & 183797.4473 \\
Ar II & 370.69 & $2 \times 10^{5}$ & 159706.5337 \\
Ar II & 375.04 & $1 \times 10^{5}$ & 159393.3850 \\
Ar II & 394.60 & $1.5 \times 10^{8}$ & 195864.7296 \\
Ar II & 400.11 & $6 \times 10^{5}$ & 172213.8798 \\
\hline
\end{tabular}

OES, optical emission spectra

the transition, $k$ is Boltzmann's constant, and $T$ is temperature. The plasma temperature can be calculated using Eq. (1) based on the line intensity ratio by measuring spectral intensity of two lines. However, the Boltzmann plot method is more accurate and reliable than the line intensity ratio method, because several spectral lines are involved with the Boltzmann method. In general, the intensity $I_{k}$ for a spectral line may be expressed s $^{23}$

$$
\ln \left[\frac{I_{k} \lambda_{k}}{g_{k} A_{k}}\right]=\ln \left(l \frac{h c}{4 \pi} \rho_{0}\right)-\frac{E_{k}}{k T}
$$

where $l$ is the path length, $h$ is Planck's constant, $c$ is light velocity, and $\rho_{0}$ is density of atoms. By plotting the left-hand term as a function of $E_{k}$, for several spectral lines, the slope $(1 / k T)$ can be evaluated (the inverse of which gives the temperature), because the first term on the right- hand side is constant and does not depend on the particular spectral line. We present in Table 1 the details of the spectral lines that we used to estimate the temperature using the Boltzmann plot method. Figure 6 shows the Boltzmann plot of emission spectra of the plasma jet produced by the plasma needle. The operating parameters are $7 \mathrm{kV}$ applied voltage, $30 \mathrm{M} \Omega$ ballast resistance, and $5 \mathrm{~mm}$ separation between the needle tip and water-covered agar-gel surface.

Table 2 shows excitation temperature calculated from OES using the Boltzmann's plot method for different applied voltage with ballast resistance of $75 \mathrm{M} \Omega$ and $5 \mathrm{~mm}$ of separation between the needle tip and water-covered agar-gel surface. We found that the relationship between varying excitation temperature and changing discharge voltages was almost negligible. It is worth noting that for a plasma needle operated with an AC power supply, excitation temperature has been reported to augment with increasing peak voltage. ${ }^{2}$ However, the present experiment revealed that the excitation temperature did not change considerably with applied voltage in the case of a plasma needle operated by a DC power supply.

Table 2 shows the variation of temperature with ballast resistance for $8 \mathrm{kV}$ of applied voltage and $5 \mathrm{~mm}$ of separation between needle tip and water-covered agar-gel 


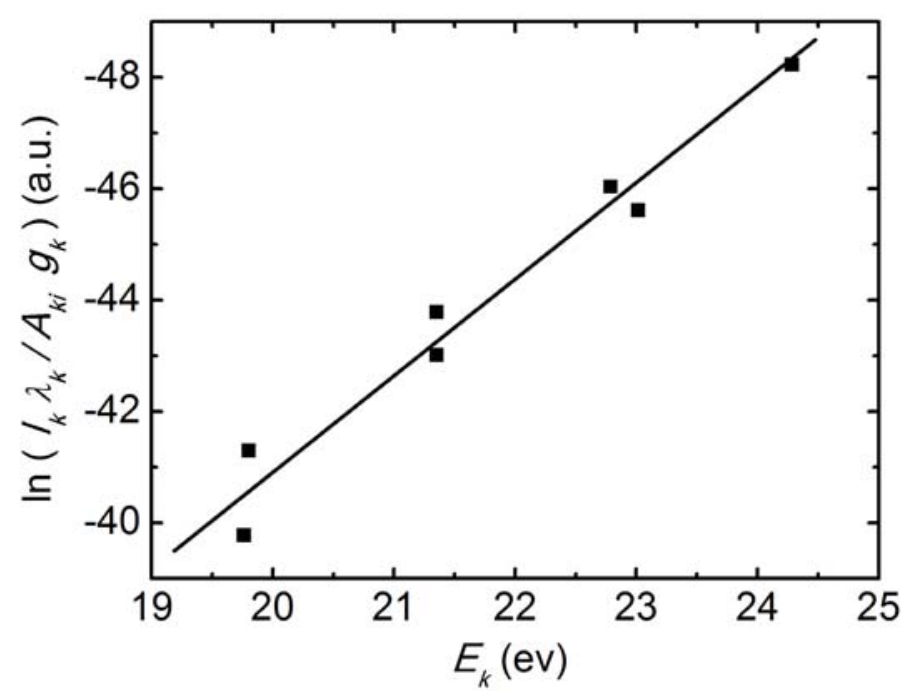

FIG. 6: Typical Boltzmann's plot for the plasma jet produced by plasma needle

TABLE 2: Estimated excitation temperature of plasma for different applied voltages, ballast resistance, and separation between the tip of the plasma needle and the water-covered agar-gel surface

\begin{tabular}{lccccc}
\hline $\begin{array}{l}\text { Applied voltage } \\
(\mathbf{k V})\end{array}$ & $\boldsymbol{T}_{\text {exc }}(\mathbf{e V})$ & $\begin{array}{c}\text { Ballast resistor } \\
(\mathbf{M} \boldsymbol{\Omega})\end{array}$ & $\boldsymbol{T}_{\text {exc }}(\mathbf{e V})$ & $\begin{array}{c}\text { Separation } \\
(\mathbf{m m})\end{array}$ & $\boldsymbol{T}_{\text {exc }}(\mathbf{e V})$ \\
\hline 7 & 0.587 & 15 & 0.5671 & 2 & 0.6878 \\
8 & 0.602 & 20 & 0.5672 & 3 & 0.6432 \\
8.5 & 0.588 & 25 & 0.5671 & 4 & 0.6045 \\
9 & 0.5994 & 30 & 0.5675 & 5 & 0.5903 \\
9.5 & 0.5866 & 35 & 0.5677 & 6 & 0.5296 \\
10 & 0.5983 & 40 & 0.5689 & 7 & 0.5285 \\
10.5 & 0.5797 & 45 & 0.5705 & 8 & 0.5221 \\
11 & 0.6027 & 50 & 0.5725 & 9 & 0.5225 \\
11.5 & 0.5948 & 55 & 0.5753 & 10 & 0.5123 \\
12 & 0.5817 & 60 & 0.5765 & 11 & 0.5122 \\
13 & 0.5778 & 65 & 0.5771 & 12 & 0.5134 \\
- & - & 75 & 0.5791 & 13 & 0.5121 \\
- & - & - & - & 14 & 0.5021 \\
- & - & - & - & 15 & 0.5043 \\
- & - & - & - & 16 & 0.5212 \\
- & - & - & - & 17 & 0.523 \\
- & - & - & - & 18 & 0.5123 \\
- & - & - & - & 19 & 0.52011 \\
- & - & - & - & 20 & 0.52012 \\
\hline
\end{tabular}


surface. Temperature augments slightly with increasing ballast resistance; however, below a threshold value of resistance, the variation in temperature is almost negligible. Table 2 also shows the temperature variation with separation between the needle tip and the water-covered agar-gel surface for $8 \mathrm{kV}$ applied voltage and $75 \mathrm{M} \Omega$ ballast resistance. We found that the variation of separation between the tip of the plasma needle and the water-covered agar-gel surface did not show any considerable change in temperature when separation was $>5 \mathrm{~mm}$. However, considerable increases in temperature occurred when the separation is $<5 \mathrm{~mm}$.

\section{CONCLUSIONS}

We used a plasma needle that was developed using a DC power source. The plasma jet produced by the plasma needle easily interacted with the human body without causing any sensation. We carried out both electrical and optical characterization of the plasma needle. It was observed that increased applied voltage augmented discharge frequency without affecting discharge voltage or discharge current. The frequency of the discharge was found to increase almost linearly with increasing applied voltage. We used the Boltzmann plot method from OES spectra to estimate excitation temperature of the plasma. In contrast to an AC plasma needle, with which the temperature increases with applied peak voltage, the temperature of the plasma jet produced by the DC plasma needle on a water-covered agar-gel surface was found to be almost independent of applied voltage. The temperature augmented with increasing ballast resistance above a threshold value of the resistance, below which the temperature was found to be almost constant. Changes in separation between the tip of the plasma needle and water-covered agar-gel surface did not show any considerable variation in temperature when separation was $>5 \mathrm{~mm}$. However, considerable increases in temperature occurred when the separation was $<5 \mathrm{~mm}$. It can thus be concluded that to apply a plasma needle with the required constant electron temperature, it is necessary to maintain a minimum separation between needle and object.

\section{ACKNOWHEGMENT}

This work was supported by the government of Chile's National Fund for Scientific and Technological Development Grant No. 11130048.

\section{REFERENCES}

1. Stoffels E, Flikweert AJ, Stoffels WW, Kroesen GMW. Plasma needle: A nondestructive atmospheric plasma source for fine surface treatment of (bio) materials. Plasma Sources Sci Technol. 2002;11:383.

2. Wu Q, Lu XP, Xiong ZL, Pan Y. A touchable pulsed air plasma plume driven by DC power supply. IEEE Trans Plasma Sci. 2010;38:12.

3. Anghel SD, Simon A, Frentiu T. Spectroscopic investigations on a low power atmospheric pressure capacitively coupled helium plasma. Plasma Sources Sci Technol. 2008;17:045016.

4. Kieft IE, Boers JLV, Caubet-Hilloutou V, Slaaf DW, Stoffels E. Electric discharge plasmas influence attachment of cultured CHO K1 cells. Bioelectromagnetics. 2004;25:362. 
5. Zhang X, Li M, Zhou R, Feng K, Yang S. Ablation of liver cancer cells in vitro by a plasma needle. Appl Phys Lett. 2008;93:021502.

6. Keidar M, Shashurin A, Volotskova O, Stepp MA, Srinivasan P, Sandler A, Trink B. Cold atmospheric plasma in cancer therapy. Phys Plasma. 2013;20:057101.

7. Lee JK, Kim MS, Byun JH, Kim KT, Kim GC, Park GY. Biomedical applications of low temperature atmospheric pressure plasmas to cancerous cell treatment and tooth bleaching. Jpn J Appl Phys. 2011;50(8S1):08JF01.

8. Park DP, Davisa K, Gilani S, Alonzo CA, Dobrynin D, Friedman G, Fridman A, Rabinovich A, Fridman G. Reactive nitrogen species produced in water by non-equilibrium plasma increase plant growth rate and nutritional yield. Curr Appl Phys. 2013;13:S19.

9. Lee EJ, Kwon JS, Uhm SH, Song DH, Kim YH, Choi EH, Kim KN. The effects of non-thermal atmospheric pressure plasma jet on cellular activity at SLA treated titanium surfaces. Curr Appl Phys. 2013;13:S36.

10. Brok WJM, Bowden MD, Dijk J, Mullen JJAM, Kroesen GMW. Numerical description of discharge characteristics of the plasma needle. J Appl Phys. 2005;98:013302.

11. Sladek REJ, Stoffels E, Walraven R, Tielbeek PJA, Koolhoven RA. Plasma treatment of dental cavities: A feasibility study. IEEE Trans Plasma Sci. 2004;32:1540.

12. Sladek REJ, Stoffels E. Deactivation of Escherichia coli by the plasma needle. J Phys D Appl Phys. 2005;38:1716.

13. Laroussi M, Mendis DA, Rosenberg M. Plasma interaction with microbes. New J Phys. 2003;5:41.

14. Kong MG, Kroesen G, Morfill G, Nosenko T, Shimizu T, Dijk J, Zimmermann LL. Plasma medicine: An introductory review. New J Phys. 2009;11:115012.

15. Lee EJ, Kwon JS, Uhm SH, Song DH, Kim YH, Choi EH, Kim KN. The effects of non-thermal atmospheric pressure plasma jet on cellular activity at SLA-treated titanium surfaces. Curr Appl Phys. 2013;13:S36.

16. Kieft IE, Laan EP, Stoffels E. Electrical and optical characterization of the plasma needle. New J Phys. 2004;6:149.

17. Kwon JS, Kim YH, Choi EH, Kim KN. The effects of non-thermal atmospheric pressure plasma jet on attachment of osteoblast. Curr Appl Phys. 2013;13:S42.

18. Setsuhara Y, Cho K, Shiratani M, Sekine M, Hori M. Plasma interactions with amino acid (1-alanine) as a basis of fundamental processes in plasma medicine. Curr Appl Phys. 2013;13:S59.

19. Lee YS, Bora B, Yap S, Wong CS, Bhuyan H, Favre M. Investigation on effect of ambient pressure in wire explosion process for synthesis of copper nanoparticles by optical emission spectroscopy. Powder Technol. 2012;222:95.

20. Lee YS, Bora B, Yap SL, Wong CS, Bhuyan H, Favre M. Understanding the mechanism of nanoparticle formation in wire explosion process. J Quant Spectrosc Radiat Trans. 2013;117:1.

21. Cho G, Lim H, Kim JH, Jin DJ, Kwon GC, Choi EH, Uhm HS. Cold plasma jets made of a syringe needle covered with a glass tube. IEEE Trans Plasma Sci. 2011;39:1234.

22. Hong Y, Lu N, Pan J, Li J, Wu Y, Shang K. Electrical and spectral characteristics of a low-temperature argon-oxygen plasma jet with syringe needle-ring electrodes. IEEE Trans Plasma Sci. 2013;41:5453.

23. Xiong Z, Huang Q, Wang Z, Lu X, Pan Y. On the electrical characteristic of atmospheric pressure Air/ $\mathrm{He} / \mathrm{O} 2 / \mathrm{N} 2 / \mathrm{Ar}$ plasma needle. IEEE Trans Plasma Sci. 2013;41:1746. 